\title{
Thermal Noise Removal From Polarimetric Sentinel-1 Data
}

\author{
Lucio Mascolo $^{\circledR}$, Member, IEEE, Juan M. Lopez-Sanchez ${ }^{\circledR}$, Senior Member, IEEE, \\ and Shane R. Cloude ${ }^{\circledR}$, Fellow, IEEE
}

\begin{abstract}
This study proposes, for the first time, an approach to remove thermal noise from the wave coherency matrix, $\mathrm{C}_{2}$, estimated from single-look complex dual-polarization Interferometric Wide Swath mode Sentinel-1 synthetic aperture radar data. The approach is straightforward; it exploits the ThermalNoiseRemoval module, provided by the European Space Agency (ESA) in its Sentinel Application Platform (SNAP) software, to remove thermal noise from the channel intensities. Then, noise correction on the complex data is applied, in order to estimate the noise-free $\mathrm{C}_{2}$ matrix. As a further novelty, the proposed approach can be implemented in SNAP, through the use of a processing graph that is here provided. The method is applied on a dense time series of Sentinel-1 data, collected on an agricultural area located near Seville, Spain. The impact of thermal noise on the estimation of the eigendecomposition parameters of $\mathbf{C}_{2}$, i.e., entropy $\left(\mathrm{H}_{2}\right)$, average alpha angle $\left(\overline{\alpha_{2}}\right)$, and anisotropy $\left(A_{2}\right)$, is assessed for different land-cover types, namely river, rice, forest, and urban areas. Monte Carlo simulations are implemented to assess the performance of the proposed approach in estimating $\mathrm{H}_{2}, \overline{\alpha_{2}}$, and $A_{2}$. Results show that the proposed noise removal method improves the estimation of these parameters for the considered land-cover classes.
\end{abstract}

Index Terms-Polarimetry, sentinel-1, thermal noise.

\section{INTRODUCTION}

$\mathbf{T}$ HERMAL noise removal is a fundamental step for a precise radiometric calibration of synthetic aperture radar (SAR) data. In Terrain Observation with Progressive Scans SAR (TOPSAR) Sentinel-1 (S1) SAR data, thermal noise consists of two additive noise sources [1], [2]. The first is related to the antenna pattern and, hence, varies in the range direction. The second is scalloping noise, which varies along the azimuth direction [1], [2].

In the case of single-look complex (SLC) coherent dualdata, polarization Interferometric Wide Swath (IW) mode S1 SAR data, the polarization state of the received wave is described by the $2 \times 2$ Hermitian and positive-semidefinite

Manuscript received September 29, 2020; revised December 10, 2020; accepted January 7, 2021. This work was supported in part by the Spanish Ministry of Science and Innovation, in part by the State Agency of Research (AEI), and in part by the European Funds for Regional Development (EFRD) under Project TEC2017-85244-C2-1-P. (Corresponding author: Lucio Mascolo.)

Lucio Mascolo and Juan M. Lopez-Sanchez are with the Institute of Computing Research (IUII), University of Alicante, 03690 Alicante, Spain (e-mail: lucio.mascolo@ua.es; juanma-lopez@ieee.org).

Shane R. Cloude is with AEL Consultants, Cupar KY15 5AA, U.K. (e-mail: aelc@mac.com).

Color versions of one or more figures in this letter are available at https://doi.org/10.1109/LGRS.2021.3050921.

Digital Object Identifier 10.1109/LGRS.2021.3050921
(PSD) wave coherency matrix $\mathbf{C}_{2}$ [3], [4]. The eigendecomposition of $\mathbf{C}_{2}$ allows obtaining key polarimetric parameters, i.e., the entropy $\left(H_{2}\right)$, the anisotropy $\left(A_{2}\right)$, and the average alpha angle $\overline{\alpha_{2}}$. It is here remarked that the physical interpretation of $H_{2}, \overline{\alpha_{2}}$, and $A_{2}$ is different from the interpretation of the eigendecomposition outputs obtained in the quadpol case. In this case, $\mathrm{H}_{2}$ represents the wave entropy, $\mathrm{A}_{2}$ represents the degree of polarization (DoP), and $\overline{\alpha_{2}}$ represents the angular separation, on the Poincaré sphere, between the polarization state of the transmitted wave and one of the received wave [3], [4].

The importance of using the full $\mathbf{C}_{2}$ matrix has been first demonstrated in [4], where the $H_{2} / \overline{\alpha_{2}}$ decomposition is applied to dual-polarized L-band ALOS -PALSAR SAR data, showing its potential for various applications such as ship detection, forest classification, and urban characterization. Furthermore, the recent studies in [5] and [6] have shown that, in the case of dual-polarization VH-VV IW S1 SAR data, the use of $\mathbf{C}_{\mathbf{2}}$, along with $\mathrm{H}_{2}, \overline{\alpha_{2}}$, and $A_{2}$, significantly improves the performance of crop-type classification and growth stages monitoring [5], and retrieval of crops biophysical parameters [6].

Due to speckle, $\mathbf{C}_{2}$ is estimated from the data by means of spatial averaging. The estimated matrix is denoted as $\widehat{\mathbf{C}_{2}}$. Therefore, $H_{2}, \overline{\alpha_{2}}$, and $A_{2}$ are estimated from the eigendecomposition of $\widehat{\mathbf{C}_{2}}$ and denoted as $\widehat{H_{2}}, \widehat{\widehat{\alpha_{2}}}$, and $\widehat{A_{2}}$, respectively. The presence of thermal noise in SLC dual-polarization IW S1 data results in a noisy $\widehat{\mathbf{C}_{2}}$ matrix. This, in turn, affects the estimation of any polarimetric parameter derived from it. Therefore, due to the potential of $\widehat{\mathbf{C}_{2}}$ in final applications [4]-[6], removing thermal noise from such a matrix represents a key step when dealing with dual-polarization IW S1 data. In such a case, an algorithm for thermal noise removal on $\widehat{\mathbf{C}_{2}}$ still lacks, with attention being paid only on removing noise from the estimated backscattering intensities (i.e., its diagonal entries). In this case, the main approach is the one provided by the European Space Agency (ESA) in [7], integrated in the Sentinel Application Platform (SNAP) software with the ThermalNoiseRemoval module. Such an approach consists of subtracting, for each polarimetric channel, the estimated noise (derived from the annotated noise vectors present in the image product files) from the single-look backscattering intensities, in order to obtain the noise-free one. Hence, even using SNAP, thermal noise removal on $\widehat{\mathbf{C}_{2}}$ is still not possible. Note that, an approach that would simply consist of applying ThermalNoiseRemoval without correcting for the off-diagonal entries 
of $\widehat{\mathbf{C}_{2}}$ would be erroneous since it would not guarantee that the matrix $\widehat{\mathbf{C}_{2}}$ is PSD. In recent years, advanced methods [1], [2], [8] have been proposed to further improve thermal noise removal on S1 cross-polarized backscattered intensity data. The method proposed in [1] is based on azimuth descalloping, noise scaling, and interswath power balancing. In [2], a method to correct the multiplicative textural noise has been proposed, whereas in [8], novel methods for scaling and power balancing and residual noise removal have been conceived. However, no algorithms have been proposed to remove thermal noise from $\widehat{\mathbf{C}_{2}}$.

In this study, we propose, for the first time, an approach for thermal noise removal on the full $\widehat{\mathbf{C}_{2}}$ matrix, derived from SLC dual-polarization IW S1 SAR data. Such a method exploits the SNAP's ThermalNoiseRemoval module. Once the noisefree intensities are obtained, these are used to apply the noise correction to the complex products that, finally, are used to obtain the noise-free $\widehat{\mathbf{C}_{2}}$ matrix. Moreover, as a further novelty, our approach can be implemented directly using only SNAP, through the use of a processing graph that is here provided. This is important since, due to the large usage of this software by all types of users, this method can be easily incorporated in current processing chains.

Finally, the proposed approach is applied on a dense time series of SLC dual-polarization VH-VV IW S1 SAR data, collected over an agricultural area located near Seville, Spain, but it can be equally applied on HH-HV data. The noisefree $\widehat{\mathbf{C}_{2}}$ matrix is obtained and, therefore, the impact of thermal noise on the estimation of $H_{2}, \overline{\alpha_{2}}$, and $A_{2}$ is assessed for different land-cover types, i.e., river, rice, forest, and urban areas. The performance of the proposed approach is evaluated by carrying out Monte Carlo (MC) simulations. The results show that when the proposed noise removal method is applied, the estimation of $H_{2}, \overline{\alpha_{2}}$, and $A_{2}$ for these landcover types improves. Regarding the estimated backscattering coefficients, the proposed approach provides the same results as the standard ThermalNoiseRemoval module in SNAP. Therefore, these observables are not analyzed in this study.

\section{Methodology}

\section{A. Noisy $\widehat{\mathbf{C}_{2}}$ Matrix}

For a given SLC XX-XY IW S1 image (with $\{X, Y\}=$ $\{H, V\}$ representing the horizontal/vertical basis), radiometric calibration allows obtaining the $\mathrm{XX}$ and $\mathrm{XY}$ complex backscattering amplitudes, affected by thermal noise, $S_{\mathrm{XX}}^{\mathrm{n}}$ and $S_{\mathrm{XY}}^{\mathrm{n}}$

$$
S_{\mathrm{XX}}^{\mathrm{n}}=\left|S_{\mathrm{XX}}^{\mathrm{n}}\right| e^{j \phi_{\mathrm{XX}}^{\mathrm{n}}}, \quad\left|S_{\mathrm{XY}}^{\mathrm{n}}\right| e^{j \phi_{\mathrm{XY}}^{\mathrm{n}}}
$$

where $\phi_{\mathrm{XX}}^{n}$ and $\phi_{\mathrm{XY}}^{n}$ denote the noisy $\mathrm{XX}$ and $\mathrm{XY}$ phase, respectively. Therefore, the noisy estimator of the wave coherency matrix, $\widehat{\mathbf{C}_{2}} \mathbf{n}$, is

$$
{\widehat{\mathbf{C}_{2}}}^{\mathbf{n}}=\left[\begin{array}{cc}
\left\langle\left|S_{\mathrm{XX}}^{\mathrm{n}}\right|^{2}\right\rangle & \left\langle S_{\mathrm{XX}}^{\mathrm{n}} S_{\mathrm{XY}}^{\mathrm{n}^{*}}\right\rangle \\
\left\langle S_{\mathrm{XY}}^{\mathrm{n}} S_{\mathrm{XX}}^{\mathrm{n}^{*}}\right\rangle & \left\langle\left|S_{\mathrm{XY}}^{\mathrm{n}}\right|^{2}\right\rangle
\end{array}\right]
$$

with $\langle\cdot\rangle$ denoting spatial averaging. Accordingly, the noisy estimators of $H_{2}, \overline{\alpha_{2}}$, and $A_{2}$ are derived from the eigendecomposition of $\widehat{\mathbf{C}_{\mathbf{2}}} \mathbf{n}$ as [3]

$$
\begin{aligned}
& {\widehat{H_{2}}}^{\mathrm{n}}=-\sum_{i=1}^{2} p_{i} \log _{2}\left(p_{i}\right), \quad \text { with } p_{i}=\frac{{\widehat{\lambda_{i}}}^{\mathrm{n}}}{{\widehat{\lambda_{1}}}^{\mathrm{n}}+{\widehat{\lambda_{2}}}^{\mathrm{n}}} \\
& {\widehat{\bar{\alpha}_{2}}}^{\mathrm{n}}=p_{1}{\widehat{\alpha_{1}}}^{\mathrm{n}}+p_{2}\left(\frac{\pi}{2}-{\widehat{\alpha_{1}}}^{\mathrm{n}}\right), \quad \text { with }{\widehat{\alpha_{1}}}^{\mathrm{n}}=\cos ^{-1}\left(\left|{\widehat{e_{1}}}^{\mathrm{n}}\right|\right) \\
& {\widehat{A_{2}}}^{\mathrm{n}}=\frac{{\widehat{\lambda_{1}}}^{\mathrm{n}}-{\widehat{\lambda_{2}}}^{\mathrm{n}}}{{\widehat{\lambda_{1}}}^{\mathrm{n}}+{\widehat{\lambda_{2}}}^{\mathrm{n}}} .
\end{aligned}
$$

In (3), ${\widehat{\lambda_{1}}}^{\mathrm{n}}$ and ${\hat{\lambda_{2}}}^{\mathrm{n}}$ are the ${\widehat{\mathbf{C}_{2}}}^{\mathbf{n}}$ eigenvalues and ${\widehat{e_{1}}}^{\mathrm{n}}$ is the first element of the eigenvector associated with the largest eigenvalue.

\section{B. Noise-Free $\widehat{\mathbf{C}_{2}}$ Matrix}

To obtain the noise-free estimator of the wave coherency matrix, referred to as $\widehat{\mathbf{C}}_{\mathbf{2}}^{\text {nf }}$, we propose a procedure consisting of the following steps.

1) Radiometric Calibration of the Noisy Complex Backscattering Amplitudes: Complex data are radiometrically calibrated to obtain $S_{\mathrm{XX}}^{\mathrm{n}}$ and $S_{\mathrm{XY}}^{\mathrm{n}}$.

2) Thermal Noise Removal on the Channel Intensities: In parallel, the digital numbers (DNs) associated with the noisy SLC data are used to obtain the intensity at the XX and the XY channels. Then, ThermalNoiseRemoval is applied to generate the noise-free intensity data. Finally, after radiometric calibration, the noise-free single-look backscattering coefficients, denoted with $\sigma_{\mathrm{XX}}^{0, \text { nf }}$ and $\sigma_{\mathrm{XY}}^{0, \text { nf }}$, are obtained.

3) Thermal Noise Removal on the Complex Backscattering Amplitudes: The noise-free complex backscattering amplitudes, $S_{\mathrm{XX}}^{\mathrm{nf}}$ and $S_{\mathrm{XY}}^{\mathrm{nf}}$, are obtained from $S_{\mathrm{XX}}^{\mathrm{n}}$ and $S_{\mathrm{XY}}^{\mathrm{n}}$, using $\sigma_{\mathrm{XX}}^{0, \mathrm{nf}}$ and $\sigma_{\mathrm{XY}}^{0, \mathrm{nf}}$ to apply the noise correction

$$
S_{\mathrm{XX}}^{\mathrm{nf}}=\sqrt{\sigma_{\mathrm{XX}}^{0, \mathrm{nf}}} e^{j \phi_{\mathrm{XX}}^{\mathrm{n}}}, \quad S_{\mathrm{XY}}^{\mathrm{nf}}=\sqrt{\sigma_{\mathrm{XY}}^{0, \mathrm{nf}}} e^{j \phi_{\mathrm{XY}}^{\mathrm{n}}} .
$$

In summary, the amplitude is taken from the square root of the noise-free backscattering coefficient, whereas the phase is retained. Here, we point out that while thermal noise does affect the phase of the polarimetric channels, the noise vectors are provided for intensity only. This is reasonable, as it is common in the literature to assume that the noise at the $\mathrm{XX}$ channel is uncorrelated from the noise at $\mathrm{XY}$ channel. Hence, since the real and the imaginary parts of each SLC channel are also assumed to be uncorrelated, this leads to uniformly distributed random phases at the SLC level (where our algorithm works). Therefore, (4) will result in correcting the real and imaginary parts of the products between the XX and XY channels, to ensure a rank-1 PSD matrix.

Then, the noise-free estimator of the wave covariance matrix is

$$
{\widehat{\mathbf{C}_{2}}}^{\mathbf{n f}}=\left[\begin{array}{cc}
\left\langle\left|S_{\mathrm{XX}}^{\mathrm{nf}}\right|^{2}\right\rangle & \left\langle S_{\mathrm{XX}}^{\mathrm{nf}} S_{\mathrm{XY}}^{\mathrm{nf}^{*}}\right\rangle \\
\left\langle S_{\mathrm{XY}}^{\mathrm{nf}} S_{\mathrm{XX}}^{\mathrm{nf}}\right\rangle & \left\langle\left|S_{\mathrm{XY}}^{\mathrm{nf}}\right|^{2}\right\rangle
\end{array}\right]
$$

Finally, the eigendecomposition of ${\widehat{\mathrm{C}_{2}}}^{\mathrm{nf}}$ provides ${\widehat{\mathrm{H}_{2}}}^{\mathrm{nf}},{\widehat{\mathrm{\alpha}_{2}}}^{\mathrm{nf}}$, and $\widehat{A_{2}}$. 


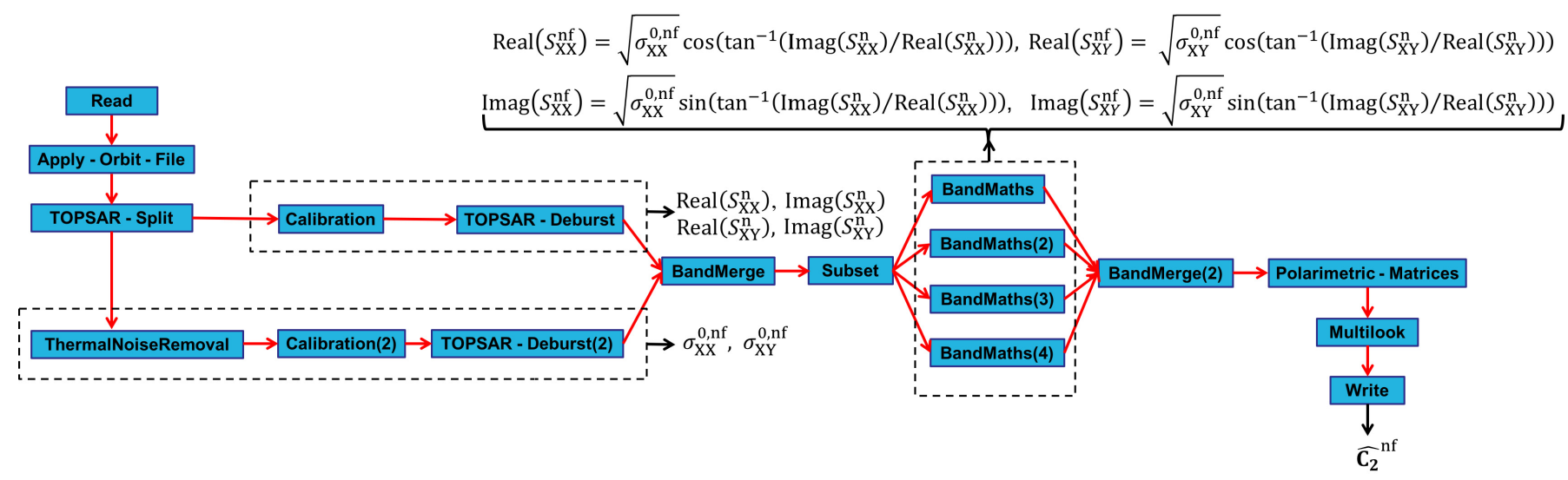

Fig. 1. Sketch of the processing graph implemented in SNAP.

\section{Noise-Induced Offsets in the}

Eigendecomposition Parameters

The presence of thermal noise in the matrix ${\widehat{\mathbf{C}_{2}}}^{\mathbf{n}}$ induces an offset in any polarimetric-based variable, including the eigendecomposition parameters. In such a case, these offsets are

$$
\begin{aligned}
\Delta \widehat{H_{2}} & ={\widehat{H_{2}}}^{\mathrm{n}}-{\widehat{H_{2}}}^{\mathrm{nf}} \\
\Delta \widehat{\overline{\alpha_{2}}} & ={\widehat{\overline{\alpha_{2}}}}^{\mathrm{n}}-{\widehat{\overline{\alpha_{2}}}}^{\mathrm{nf}} \\
\Delta \widehat{A_{2}} & =\left|{\widehat{A_{2}}}^{\mathrm{n}}-{\widehat{A_{2}}}^{\mathrm{nf}}\right| .
\end{aligned}
$$

Then, since $\widehat{\mathbf{C}_{2}} \mathbf{n}$ is characterized by higher depolarization, we expect, on the average, $\Delta \widehat{H_{2}}>0, \Delta \widehat{\widehat{\alpha_{2}}}>0^{\circ}$, and $\Delta \widehat{A_{2}}>0$.

\section{Implementation in SNAP}

The proposed approach can be implemented in SNAP, using the processing graph (.xml file) shown in Fig. 1 (obtained with the GraphBuilder tool). Such a graph is provided in the GitHub repository available online at [https://github.com/LucioMascolo/Noise-free-C2-graph].

Initially, for a given SLC XX-XY IW S1 image, the ApplyOrbit-File (which allows applying the precise orbit file of the image) and the TOPSAR-Split (which allows selecting the subswath and the bursts of interest) modules are applied. Then, the processing chain splits into two branches. In the upper branch, complex data are radiometrically calibrated (Calibration module) and then debursted (TOPSAR-Deburst module), in order to obtain the real and the imaginary parts of the noisy complex backscattering amplitudes. In the lower branch, the ThermalNoiseRemoval module is applied to remove noise from the channel intensities. The output products are radiometrically calibrated and then debursted, in order to obtain the single-look backscattering coefficients $\sigma_{\mathrm{XX}}^{0, \text { nf }}$ and $\sigma_{\mathrm{XY}}^{0, \mathrm{nf}}$. The resulting products of these two branches are merged through the BandMerge operator and, then, optionally the Subset operator is applied, in order to extract only an excerpt of the imaged scene. As a third step, (4) is applied using the BandMaths operator: BandMaths and BandMaths(2) provide the real and imaginary parts of $S_{\mathrm{XX}}^{\mathrm{nf}}$ and BandMaths(3) and BandMaths(4) provide the real and imaginary parts of $S_{\mathrm{XY}}^{\mathrm{nf}}$.

These output products are then merged to serve as input for the Polarimetric-Matrices module, where SNAP creates a single look $2 \times 2$ wave coherency matrix. Finally, ${\widehat{\mathbf{C}_{2}}}^{\text {nf }}$ is obtained with the Multilook module and then saved as image product file (Write operator).

\section{EXPERIMENTS}

\section{A. Test Site and Data Processing}

The proposed noise removal method is applied on an agricultural test site, located near Seville, Spain, where rice is the main crop. This area has been considered in previous literature studies, e.g., in [9] for crop-type mapping using S1 interferometric coherence data. Apart from rice and other crops, different land-cover types are present, including the Guadalquivir river, forests, and urban areas.

The SAR data consist of a time series with 32 SLC dualpolarization VH-VV IW S1 images, collected by both S1A and S1B sensors (i.e., six-day revisit time, with relative orbit: 74 , ascending pass), acquired from May 28 to November 30. Such a time series is processed in SNAP version 7 , where two processing chains are implemented, both using the batch processing module. The first chain is aimed at obtaining ${\widehat{\mathbf{C}_{2}}}^{\text {nf }}$, by applying the processing graph in Fig. 1. The area of interest is covered by subswath IW1, which is selected in the TOPSAR-Split module, along with the bursts (bursts 3-5 for S1A and bursts 7-9 for S1B). In this case, the Subset operator is used to select only a region of interest (ROI) from the debursted data. After noise correction, the noisefree matrix $\widehat{\mathbf{C}}_{\mathbf{2}}^{\mathbf{n f}}$ is estimated by a $4 \times 1$ (range $\times$ azimuth) multilook, which provides a square pixel on the ground of about $15 \mathrm{~m}$. Then, further processing is applied: 1) the intensity-driven adaptive-neighborhood (IDAN) speckle filter [10] is applied on each $\widehat{\mathbf{C}}_{\mathbf{2}}{ }^{\text {nf }}$ matrix (setting number of looks $=1$ and adaptive neighborhood size $=11)$ and 2) each $\widehat{\mathbf{C}}_{\mathbf{2}}^{\text {nf }}$ matrix is geocoded, resampling the output products at $10-\mathrm{m}$ pixel spacing. As a final step, the geocoded $\widehat{\mathbf{C}_{2}}$ mf matrices are coregistered by using the Create Stack module, to have them in the same geographical grid. In Fig. 2(a), a Google Earth picture of the test site is shown, with the extent of the extracted ROI highlighted in white. Fig. 2(b) shows a falsecolor RGB composite, with the Red, Green, and Blue channels associated with $\widehat{H}_{2}^{\text {nf }}$ collected on June 15 , August 14, and October 1, respectively. The second processing chain consists 


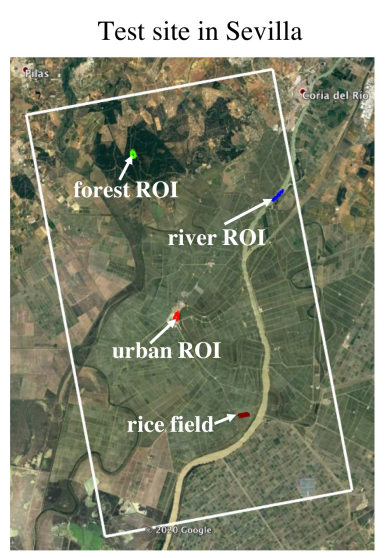

(a)

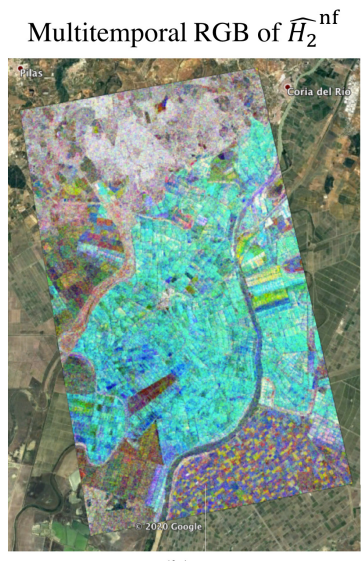

(b)
Fig. 2. (a) Google Earth picture of the Seville test site, where the excerpt of the $\mathrm{S} 1$ scene is highlighted in white. The polygons corresponding to the water, rice, forest, and urban ROIs are also visible. (b) False-color RGB composite obtained from the $\widehat{H}_{2}$ nf time series, where the Red, the Green, and the Blue channel are associated with the images collected on June 15, August 14, and October 1 , respectively.

of the "classical" estimation approach, aimed at obtaining the time series of the noisy ${\widehat{\mathbf{C}_{2}}}^{\mathbf{n}}$ matrices. The same settings for the multilook, speckle filtering, and geocoding steps are used, but thermal noise removal is not performed.

\section{B. Impact of Thermal Noise on $\widehat{H_{2}}, \widehat{\overline{\alpha_{2}}}$, and $\widehat{A_{2}}$}

In order to analyze the influence of thermal noise on the estimation of the eigendecomposition parameters, four ROIs, corresponding to different land-cover types, are considered: an excerpt of the Guadalquivir river, a rice field, a forest area, and an urban area (an excerpt of the town of Isla Mayor). The polygons of these ROIs are overlaid on the Google Earth picture of Fig. 2(a). For each land-cover class, ${\widehat{H_{2}}}^{\mathrm{nf}},{\widehat{\overline{\alpha_{2}}}}^{\mathrm{nf}}$, and ${\widehat{A_{2}}}^{\text {nf }}$ are estimated from $\widehat{\mathbf{C}}_{2}^{\text {nf }}$, whereas ${\widehat{H_{2}}}^{\mathrm{n}},{\widehat{\alpha_{2}}}^{\mathrm{n}}$, and ${\widehat{A_{2}}}^{\mathrm{n}}$ are estimated from $\widehat{\mathbf{C}}_{2}$. Then, (6) is applied to obtain $\Delta \widehat{H}_{2}, \Delta \widehat{\widehat{\alpha_{2}}}$, and $\Delta \widehat{A_{2}}$. The average values of $\widehat{H}_{2}$ nf,${\widehat{\alpha_{2}}}^{\text {nf }}$ and ${\widehat{A_{2}}}^{\text {nf }}$ are shown in Fig. 3(a), (c), and (e), respectively, while the average $\Delta \widehat{H_{2}}$, $\Delta \widehat{\overline{\alpha_{2}}}$, and $\Delta \widehat{A_{2}}$ values are shown in Fig. 3(b), (d), and (f), respectively. All the parameters are plotted against the Day of the Year (DoY).

The behavior of the estimated eigendecomposition parameters is the one expected for these land-cover types. For the river and the rice classes, the large dynamic range of $\widehat{H}_{2}^{\text {nf }}$, ${\widehat{\alpha_{2}}}^{\mathrm{nf}}$, and ${\widehat{A_{2}}}^{\mathrm{nf}}$ induces large temporal variations in the offsets. In the case of the river, the polarimetric parameters depend on the state of the water surface, which is driven by wind conditions, river's currents, etc. Regarding rice, ${\widehat{H_{2}}}^{n f},{\widehat{\overline{\alpha_{2}}}}^{n f}$, and $\widehat{A}_{2}^{\text {nf }}$ vary according to its phenological stages. It can be noted that when weak depolarization is experienced, i.e., for the river in most of the cases and for the rice field at the beginning of the growing season (between DoY 154 and DoY 190), the offsets exhibit larger values. $\Delta \widehat{H}_{2}$ is $\sim 0.1$, while $\Delta \widehat{\overline{\alpha_{2}}}\left(\Delta \widehat{A_{2}}\right)$ ranges between $\sim 3^{\circ}$ and $\sim 5^{\circ}$ (between $\sim 0.05$ and $\sim 0.007)$. However, while it is easier to relate $\Delta \widehat{H}_{2}$ to changes in $\widehat{H}_{2}^{\text {nf }}$, it is hard to recognize a clear trend in $\Delta \widehat{\overline{\alpha_{2}}}$ and $\Delta \widehat{A_{2}}$. On the other hand, when moderate-to-high

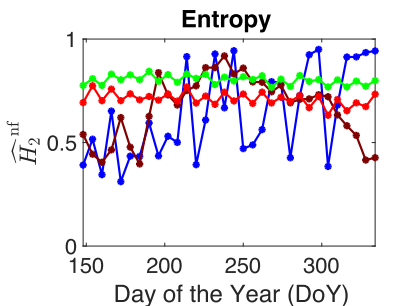

(a)

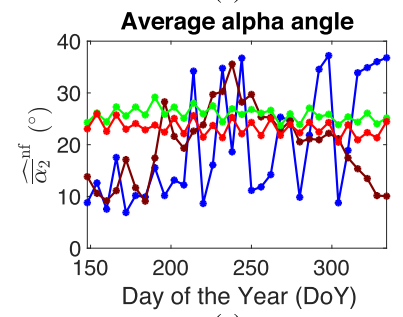

(c)

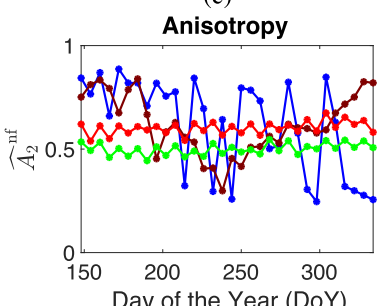

(e)

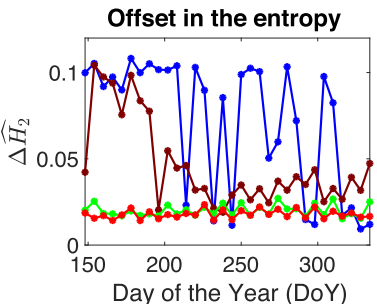

(b)

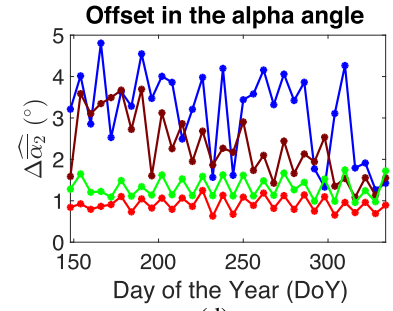

(d)

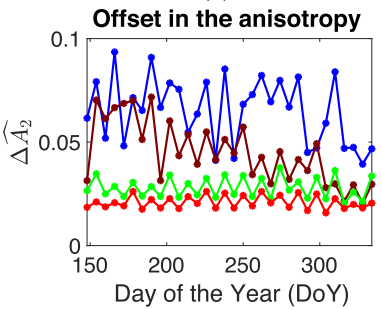

(f)
Fig. 3. (a), (c), and (e) Evolution of ${\widehat{H_{2}}}^{\text {nf }}, \widehat{\widehat{\alpha}}_{2}^{\text {nf }}$ and ${\widehat{A_{2}}}^{\text {nf }}$ for each land-cover type, respectively. (b), (d), and (f) Evolution of the noise-induced offsets.

depolarization is in place, the offsets are lower, indicating a less significant impact of thermal noise. For the river, this occurs at some acquisition dates, e.g., at DoY 232 and DoY 244 , where $\Delta \widehat{H_{2}}$ is $\sim 0.01, \Delta \widehat{\overline{\alpha_{2}}}$ is $\sim 1^{\circ}$, and $\Delta \widehat{A_{2}}$ is $\sim 0.04$ For the rice field, higher depolarization is observed as plants develop, from DoY 202 onward. Accordingly, the lower $\Delta \widehat{H_{2}}$, $\Delta \widehat{\overline{\alpha_{2}}}$, and $\Delta \widehat{A_{2}}$ values range from $\sim 0.02$ to $\sim 0.05$, from $\sim 1.5^{\circ}$ to $\sim 3^{\circ}$, and from $\sim 0.03$ to $\sim 0.05$, respectively.

Regarding the forest and urban area, a moderate-to-high depolarization, stable along time, is observed, resulting in a low impact of thermal noise in the estimation. Accordingly, the low $\Delta \widehat{H_{2}}, \Delta \widehat{\overline{\alpha_{2}}}$, and $\Delta \widehat{A_{2}}$ values are stable around $\sim$ $0.02, \sim 1^{\circ}$, and $\sim 0.025$, respectively. Moreover, the apparent sawtooth behavior, more pronounced for these two classes, is likely due to the difference between the S1A and S1B backscattering coefficients and noise levels [11].

\section{Performance Assessment With MC Simulations}

The performance of the proposed approach is evaluated by implementing, for each land-cover type, MC simulations, using the procedure proposed in [12]. For these simulations, the $\widehat{\mathbf{C}_{2}}$ nf matrix relevant to the S1 acquisition on July 21 (DoY 202) is used.

The core idea is to assume that, for a large number of looks $\left(N_{L}\right), \widehat{\mathbf{C}}_{\mathbf{2}} \mathbf{n f}$ represents the true wave coherency matrix, $\mathbf{C}_{\mathbf{2}}$; ${\widehat{H_{2}}}^{\mathrm{nf}},{\widehat{\alpha_{2}}}^{\mathrm{nf}}$, and ${\widehat{A_{2}}}^{\mathrm{nf}}$ represent the true parameters, $H_{2}, \overline{\alpha_{2}}$, and $A_{2}$. This is in line with the analysis carried out in [13].

The first $\mathrm{MC}$ simulation is aimed at simulating $\mathbf{C}_{2}$. For each land-cover ROI, $\widehat{\mathbf{C}}_{\mathbf{2}}^{\text {nf }}$ is averaged over all the pixels. 
TABLE I

RMSE OF THE NOISY ESTIMATORS FOR EACH LAND-COVER CLASS

\begin{tabular}{c|c|c|c}
\hline & $\operatorname{RMSE}\left({\widehat{H_{2}}}^{\mathrm{n}}\right)$ & $\operatorname{RMSE}\left({\widehat{\bar{\alpha}_{2}}}^{\mathrm{n}}\right)\left({ }^{\circ}\right)$ & $\operatorname{RMSE}\left({\widehat{A_{2}}}^{\mathrm{n}}\right)$ \\
\hline River & 0.23 & 9 & 0.2 \\
\hline Rice & $7 \times 10^{-2}$ & 3.9 & $9 \times 10^{-2}$ \\
\hline Forest & $2.4 \times 10^{-2}$ & 1.6 & $3.6 \times 10^{-2}$ \\
\hline Urban & $1.5 \times 10^{-2}$ & 0.9 & $1.7 \times 10^{-2}$ \\
\hline
\end{tabular}

TABLE II

RMSE OF THE NOISE-FREE ESTIMATORS FOR EACH LAND-COVER CLASS

\begin{tabular}{c|c|c|c}
\hline & $\operatorname{RMSE}\left({\widehat{H_{2}}}^{\mathrm{nf}}\right)$ & $\operatorname{RMSE}\left({\widehat{\bar{\alpha}_{2}}}^{\mathrm{nf}}\right)(\circ)$ & $\operatorname{RMSE}\left({\widehat{A_{2}}}^{\mathrm{nf}}\right)$ \\
\hline River & $10^{-4}$ & 0.13 & $10^{-4}$ \\
\hline Rice & $2 \times 10^{-4}$ & 0.15 & $2 \times 10^{-4}$ \\
\hline Forest & $4 \times 10^{-4}$ & 0.15 & $6 \times 10^{-4}$ \\
\hline Urban & $1.6 \times 10^{-3}$ & 0.1 & $1.7 \times 10^{-3}$ \\
\hline
\end{tabular}

This average matrix is used as an input to obtain $\mathbf{C}_{2}$, setting $N_{L}=10000$. Finally, the eigendecomposition of $\mathbf{C}_{2}$ provides the true $\mathrm{H}_{2}, \overline{\alpha_{2}}$, and $A_{2}$. Then, in order to simulate, say $N_{M}$, realizations of the noisy and noise-free estimators, $N_{M}$ MC simulations (with $N_{M}=10000$ ) are implemented, using $\mathbf{C}_{2}$ as an input. At each iteration, the "classical" estimation approach, i.e., $\widehat{\mathbf{C}_{2}} \mathbf{n}$, is first simulated. The VH and VV noise intensities (obtained by subtracting the single-look noise-free backscattering coefficients from the noisy ones) are added on the simulated true complex scattering amplitudes, thus simulating the noisy SLC data. The latter allows obtaining ${\widehat{\mathbf{C}_{2}}}^{\mathbf{n}}$, from which ${\widehat{H_{2}}}^{\mathrm{n}},{\widehat{\overline{\alpha_{2}}}}^{\mathrm{n}}$, and ${\widehat{\mathrm{A}_{2}}}^{\mathrm{n}}$ are derived. Then, the proposed approach is applied to remove thermal noise from the simulated noisy SLC data. After noise correction, $\widehat{\mathbf{C}}_{\mathbf{2}}^{\text {nf }}$ is obtained, along with ${\widehat{H_{2}}}^{\mathrm{nf}},{\widehat{\bar{\alpha}_{2}}}^{\mathrm{nf}}$, and ${\widehat{A_{2}}}^{\mathrm{nf}}$.

Finally, the performances of these estimators are evaluated by the root mean square error (RMSE)

$$
\operatorname{RMSE}(\widehat{\theta})=\sqrt{\operatorname{var}(\widehat{\theta})+b^{2}}
$$

where $\widehat{\theta}$ is an estimator of the true parameter $\theta, \operatorname{var}(\cdot)$ denotes the variance of an estimator, and $b=E(\widehat{\theta})-\theta$ is the bias, with $E(\cdot)$ denoting statistical expectation.

For each land-cover type, the RMSE for the noisy and noise-free estimators are listed in Tables I and II, respectively. By comparing these two tables, one can clearly see that, for all the classes, the estimation of $H_{2}, \overline{\alpha_{2}}$, and $A_{2}$ is improved when the proposed noise removal approach is applied. For $\mathrm{H}_{2}$ and $\mathrm{A}_{2}$, the difference, in terms of RMSE, between the noise-free estimators and the noisy ones is: three orders of magnitude for the river class, $\sim 2$ orders of magnitude for both rice and forest class, and one order of magnitude for the urban class. In the case of $\overline{\alpha_{2}}$, such differences are: almost two orders of magnitude for the river class, more than one order of magnitude for rice class, one order of magnitude for the forest class, and about one order of magnitude for the urban class.

\section{CONCLUSION}

For the first time, a method to remove thermal noise from the $\mathbf{C}_{2}$ matrix, estimated from SLC dual-polarization IW
S1 SAR data, is proposed in this study. Such an approach exploits SNAP's ThermalNoiseRemoval module and provides ${\widehat{\mathbf{C}_{2}}}^{\text {nf }}$ in a straightforward and effective way. As a further novelty, we provide a processing graph, which allows implementing the approach using only SNAP. This represents a significant advantage for a wide range of SNAP users.

The proposed method is applied on a dense time series of SLC dual-polarization VH-VV IW S1 SAR data and tested on different land-cover types, i.e., river, rice, forest, and urban areas. The time series of $\Delta \widehat{H_{2}}, \Delta \widehat{\overline{\alpha_{2}}}$, and $\Delta \widehat{A_{2}}$ for these classes allows evaluating the impact of thermal noise on the estimation of the eigendecomposition parameters.

Furthermore, for each class, we carried out MC simulations to compare the performances of the noisy estimators, i.e., the ones obtained from the "classical" $\mathbf{C}_{2}$ estimation, with the noise-free ones. The resulting RMSEs show that the proposed noise removal approach improves the estimation of the eigendecomposition parameters for the considered landcover classes.

In conclusion, the proposed approach allows improving polarimetric analysis undertaken with dual-polarization S1 data, and hence, it is expected to be important for final applications, including the ones in [4]-[6].

\section{REFERENCES}

[1] J.-W. Park, A. A. Korosov, M. Babiker, S. Sandven, and J.-S. Won, "Efficient thermal noise removal for Sentinel-1 TOPSAR crosspolarization channel," IEEE Trans. Geosci. Remote Sens., vol. 56, no. 3, pp. 1555-1565, Mar. 2018.

[2] J.-W. Park, J.-S. Won, A. A. Korosov, M. Babiker, and N. Miranda, "Textural noise correction for Sentinel-1 TOPSAR cross-polarization channel images," IEEE Trans. Geosci. Remote Sens., vol. 57, no. 6, pp. 4040-4049, Jun. 2019.

[3] S. R. Cloude, Polarisation. Applications in Remote Sensing. London, U.K.: Oxford Univ. Press, 2009.

[4] S. R. Cloude, "The dual polarisation entropy alpha decomposition: A PALSAR case study," in Proc. POLINSAR, Frascati, Italy, Mar. 2007, p. 2.

[5] Z.-S. Zhou et al., "Preparation of analysis ready polsar data for the australian geoscience data cube," in Proc. IEEE Int. Geosci. Remote Sens. Symp. (IGARSS), Jul. 2017, pp. 5229-5232, doi: 10.1109/IGARSS. 2017.8128180.

[6] D. Mandal et al., "Dual polarimetric radar vegetation index for crop growth monitoring using sentinel-1 SAR data," Remote Sens. Environ., vol. 247, Sep. 2020, Art. no. 111954.

[7] Thermal Denoising of Products Generated by the S-1 IPF. ESA document. Accessed: Sep. 24, 2020. [Online]. Available: https://sentinel.esa.int/documents/247904/2142675/Thermal-Denoisingof-Products-Generated-by-Sentinel-1-IPF.pdf

[8] Y. Sun and X.-M. Li, "Denoising Sentinel-1 extra-wide mode crosspolarization images over sea ice," IEEE Trans. Geosci. Remote Sens., early access, Jul. 9, 2020, doi: 10.1109/TGRS.2020.3005831.

[9] A. Mestre-Quereda, J. M. Lopez-Sanchez, F. Vicente-Guijalba, A. W. Jacob, and M. E. Engdahl, "Time-series of Sentinel-1 interferometric coherence and backscatter for crop-type mapping," IEEE J. Sel. Topics Appl. Earth Observ. Remote Sens., vol. 13, pp. 4070-4084, 2020.

[10] G. Vasile, E. Trouve, J. S. Lee, and V. Buzuloiu, "Intensity-driven adaptive-neighborhood technique for polarimetric and interferometric SAR parameters estimation," IEEE Trans. Geosci. Remote Sens., vol. 44, no. 6, pp. 2695-2709, Jun. 2006.

[11] G. Schmidt, M. Schwerdt, N. Miranda, and J. Reimann, "Radiometric comparison within the Sentinel-1 SAR constellation over a wide backscatter range," Remote Sens., vol. 12, no. 10, pp. 854-873, Jan. 2020.

[12] J. Lee and E. Pottier, Polarimetric Radar Imaging: From Basics to Applications. Boca Raton, FL, USA: CRC Press, 2009.

[13] C. Lopez-Martinez, E. Pottier, and S. R. Cloude, "Statistical assessment of eigenvector-based target decomposition theorems in radar polarimetry," IEEE Trans. Geosci. Remote Sens., vol. 43, no. 9, pp. 2058-2074, Sep. 2005. 